\title{
Energy balance and its relationship with metabolic disease in Bangladeshi middle-aged women
}

\author{
Malik Syeda Umme Fahmida ${ }^{1 *}$, Begum Musammat Kulsuma ${ }^{2}$, Ahmad Abu Toha Reza ${ }^{3}$ \\ 1, 2 Shahjalal University of Science and Technology, Sylhet, Bangladesh \\ ${ }^{3}$ Shahjalal University of Science and Technology, Sylhet, Bangladesh
}

Key Words:

Body mass index

Physical activity

Energy intake

Received: 15 May 2016

Accepted: 29 May 2016

Published: 24 June 2016

\begin{abstract}
Obesity has been rising as a major public health challenge. The obesity epidemic is more in middle-aged women in urban areas than those in rural areas. The metabolic disease has characteristic features like a higher prevalence of abdominal obesity in South Asians than white Caucasians. Because of the occurrence of metabolic syndrome at a lower range of Body Mass Index (BMI) and it is proposed that cut-offs for both measures of obesity should be changed to reach a new set point for overweight BMI $23-24.9 \mathrm{~kg} / \mathrm{m} 2$ and $\geq 25 \mathrm{~kg} / \mathrm{m} 2$ for obesity for South Asians. The strength and direction of energy balance (energy flux and body weight) and type 2 diabetes (T2D) development have not been adequately investigated. In this study, nearly 2000 middle-aged Bangladeshi women were taken as a study population who had not suffered from diabetes. These women had to face interviews at the initial phase, and finally, repeated investigation was done regularly to collect information on their metabolic risk factors. Throughout 3.6 years follow-up, 850 new cases of T2D were documented. During follow-up, 850 new cases of T2D were documented. Energy Intake (EI) was associated with increased risk, and energy expenditure with Physical Activity (PA) was associated with weight change and decreased risk of T2D. In our population, we found women tend to develop T2D who are less active with higher EI than those more active with lower EI. We also observed the association was more evident among overweight and obese women (BMI $\geq 25 \mathrm{~kg} / \mathrm{m} 2$ ). These follow-up studies suggest that energy balance plays a dominant role in the development of weight regulation, and this effect may be modified by BMI. Bodyweight regulation is a complex collection and genetic variable that control energy intake and expenditure. Further study of this concept may help to achieve a healthy weight.
\end{abstract}

(C) 2016 The Author(s). Published by TAF Publishing.

\begin{abstract}
INTRODUCTION
The increasing prevalence of metabolic diseases in numerous populations throughout the world is currently a huge global health problem. Obesity and weight change are multifactorial and considered to be a result of either excessive caloric intake with the significant influence of genetics or insufficient physical activity. There is a debate about that food restriction alone will not be effective in reducing obesity if the existence of metabolic inefficiency is increased. Despite the normal physiology of a control system, the activity levels of most people decreased dramatically over a period. Theoretically, an individual can achieve energy balance in multiple ways. In many cases, genetic polymorphisms identified leading to obesity, but is not well worked out. The idea that energy balance is best regulated at a high level of physical activity was first proposed by Mayer and colleagues in the 1950s [1].
\end{abstract}

\footnotetext{
* Corresponding author: Malik Syeda Umme Fahmida

†Email: fahmidareza@yahoo.com
} 
Research groups are beginning to focus on the physiological, medical, and public health impact of sitting too much. There is enough information about exercise physiology to support the well-documented public health guidelines promoting at least $150 \mathrm{~min} /$ week of moderate-vigorous leisure-time physical activity aimed at decreasing risks for metabolic diseases $[2,3]$.

The decline in daily activity that came from industrialization, mechanical, transportation, urbanization, and other aspects of technology created the largest decline in activity and created the right conditions under which over feeding, widespread availability, and availability of high fat diet could have a major impact on body weight. On the activity front, as reduced physical activity levels, body weight increased which would have an impact on positive energy balance [4]. The theoretical framework to explain weight change and obesity is currently based on the concept of energy balance that energy can be converted among forms but must be conserved [5].

Body weight regulation is a complex collection of physiologic, metabolic, environmental, behavioral, and genetic factors that control active people consume more kcal/day. Furthermore, it has been suggested that by reducing positive energy balance by $45-100 \mathrm{kcal} /$ day, weight gain in $90 \%$ of the adult population could be prevented [6]. A number of animal models have identified specific mechanisms such as a reduced number of insulin receptors have been suggested as the mechanisms behind the obesity. If such mechanisms exist in humans, that could be either a cause or consequence of obesity [7]. According to the result of National Health and Nutrition Examination Survey (NHANES) taken from 1971 to 2000, we can say that the average energy intake increased on a regular basis [8]. The present study aims to outline how the development of central appetite and expenditure circuits and peripheral metabolic tissues are likely to play a key role in the long-term regulation of energy balance.

\section{LITERATURE REVIEW}

It is widely accepted that industrialization has had a profound impact on everyday life. There has been a shift from manual work to service jobs. Substantial research is directed and discussed how to help to address obesity. Research investigation of Chinese adults identified an inverse relationship between occupational and domestic physical activity patterns and body weight. A better understanding from recent Canadian study found that the ownership of sedentariness (e.g. television, car and computer) increased the likelihood of obesity and diabetes, and this was mediated in part by effects on physical activity, sitting time and dietary energy intake [9]. Metabolic syndrome is a constellation of risk factors for CVD and type 2 diabetes including plasma triglyceride, HDL cholesterol, plasma glucose, blood pressure, and central adiposity. Studies have shown that the classification of people with metabolic syndrome and related metabolic risk factors, excessive adiposity or weight gain, poor glucose management in children with type 1 diabetes, and type 2 diabetes risk have all been directly related to sitting time and/or inversely to low nonexercise activity [10-11]. It is important to note that, in physiological terms, the energy cost of weight gain is substantially greater than the energy cost of weight loss [12]. In addition, experimental studies such as the classic overfeeding and exercising studies usually imposed large energy imbalances over a relatively short period of time is not acceptable and the physiologic responses to large imbalances may be prominent and mask the true equilibrium status [9]. Obesity and weight change are influenced by genetics, environmental factors, physiology, behavior, and interactions of these variables [13]. Numerous studies have shown a compensatory response to reduce energy intake resulting in reduced 
physical activity and caloric expenditure [14]. Epigenetics study also receiving greater attention in term of metabolic diseases [15].

\section{RESEARCH MODEL}

All measurements were obtained by trained research staff who had demonstrated competency and were certified in the specific measurement technique. Baseline 1 began with an extensive review of medical history and demographic information. Baseline 2 included measurements of resting BP, height and weight as well as a maximal fitness test using ECG and BP measurement. Baseline 3 was conducted while the participant was in a 12-hr fasting state and 24-hr abstention of physical activity. During the follow-up period, the participants received three questionnaires about dietary history on random days. These surveys included extensive queries about dietary intake over the previous 24-hr period and the surveys were conducted by licensed nutritionist who had experience in conducting dietary assessments. Body weight was the dependent variable of interest with TEE being the main independent variable of interest and height, age, and physical activity as confounding variables.

\section{Energy Intake (Random 24-hr dietary Recalls)}

During the baseline dietary assessment period, approximately $75 \%$ of participants completed three dietary assessment and more than $97 \%$ of participants completed at least two dietary recall interviews. Energy intake averaged $2186.29 \pm 178.884 \mathrm{kcals} /$ day for obese women and $1870.99 \pm 199.745 \mathrm{kcals} /$ day for non-obese women with the largest component of those calories coming from carbohydrates.

\section{Energy Expenditure (Activity monitors)}

Total daily energy expenditure for obese and non-obese women were $2063.15 \pm 165.955$ kcals/day and $1980.60 \pm 202.501 \mathrm{kcals} /$ day respectively. Approximately half of the calories expended by both groups during sedentary activity. The term energy gap was coined to estimate the degree of change in the energy balance required for success in body weight goals [8].

\section{Analytical Approach}

The primary aim of the EBS is to investigate how energy intake and energy expenditure are related to changes in body weight over time. Changes in body weight depend on changes in energy intake and expenditure as well as body weight at the start of the study period [16]. The energy balance study has multiple outcomes including body weight, body composition, and cardio metabolic variables. So, the associations between changes in energy intake, expenditure, body weight and other outcomes should be consistent across all analyses.

\section{DATA ANALYSIS}

A total of 2000 participants in follow-up for 3.6 years were taken. Based on the inclusion and exclusion criteria 850 participants completed all baseline sessions and included in the dataset. The mean age was $50.81 \pm 14.761$. The characteristics of the 850 participants were shown in Table 1. Biochemical characteristics were shown in Table 2. The relation between obese and non-obese groups of this study were shown in Table 3. From this table significant difference was found in all variable. From biochemical parameters shown in table 4, we found significant difference only on TG between obese and non-obese groups. 
As BMI is a very strong risk factor for the development of IR, we present associations between components of energy balance stratified by BMI categories in Table 4. Most mean values were attained statistical significance. BMI and standardized weight gain were associated with a higher risk of IR given in Table 5. Significant difference was found among all parameters. Participants with a higher BMI were more likely to be obese and insulin resistance. The level of significance is indicated by $p$ value $<0.05$ (significance). Change in age related insulin resistance was shown in Figure 1. Among three age groups (Group1: 20-40 yrs.; Group 2: 41-60 yrs.; Group 3: 61-80yrs) group 2 is more significantly raised by metabolic factors.

TABLE 1. Subject characteristics at baseline

\begin{tabular}{llll}
\hline \hline Variable & Mean \pm SD & $\boldsymbol{t}$-value & $\boldsymbol{p}$-value \\
\hline Age & $50.81 \pm 14.761$ & -4.2950418 & 0.0000197 \\
Height & $155.36 \pm 4.845$ & -18.6658178 & 0.00 \\
Weight & $59.49 \pm 6.082$ & -14.2466737 & 0.00 \\
BMI & $25.94 \pm 2.533$ & -1.7412883 & 0.0820 \\
Sys & $122.58 \pm 12.181$ & -3.6394095 & 0.0003 \\
Dias & $63.29 \pm 7.677$ & -4.8224592 & 0.00 \\
EI & $2000.68 \pm 230.561$ & -11.4303160 & 0.00 \\
EE & $1954.48 \pm 174.654$ & -14.2977344 & 0.00 \\
\hline \hline
\end{tabular}

TABLE 2. Biochemical characteristics in study subjects

\begin{tabular}{llll}
\hline \hline Variable & Mean \pm SD & $\boldsymbol{t}$-value & $\boldsymbol{p}$-value \\
\hline TC & $197.91 \pm 59.207$ & 5.3800961 & 0.00 \\
HDL & $37.75 \pm 10.504$ & 2.7387699 & 0.0063 \\
LDL & $126.02 \pm 48.525$ & 4.8772265 & 0.00 \\
TG & $197.59 \pm 118.158$ & 0.7980835 & 0.4251 \\
Non-HDL & $160.16 \pm 57.401$ & 4.9741188 & 0.00 \\
TC: HDL & $5.57 \pm 2.113$ & 1.5239708 & 0.1280 \\
LDL: HDL & $3.55 \pm 1.632$ & 2.2542174 & 0.0245 \\
TG: HDL & $5.95 \pm 4.984$ & -1.0267927 & 0.3049 \\
FBS & $6.67 \pm 2.675$ & -0.1147886 & 0.9086 \\
IR & $3.48 \pm 2.715$ & 0.0320984 & 0.9744 \\
\hline \hline
\end{tabular}

TABLE 3. The anthropometric data of obese and non-obese groups

\begin{tabular}{lllll}
\hline \hline Variable & Obese & Non-obese & $\boldsymbol{t}$-value & $\boldsymbol{p}$-value \\
\hline Age & $54.71 \pm 14.161$ & $49.36 \pm 15.634$ & -4.4801443 & 0.00 \\
Height & $158.56 \pm 6.112$ & $159.84 \pm 6.958$ & 2.4260382 & 0.0157 \\
Weight & $64.98 \pm 6.063$ & $57.60 \pm 6.597$ & -14.5815289 & 0.00 \\
BMI & $27.34 \pm 1.972$ & $23.25 \pm 1.099$ & -36.6236294 & 0.00 \\
Sys & $125.07 \pm 10.798$ & $122.13 \pm 14.963$ & -2.7037835 & 0.0072 \\
Dias & $65.24 \pm 7.983$ & $63.43 \pm 8.490$ & -2.7703447 & 0.0059 \\
EI & $2186.29 \pm 178.884$ & $1870.99 \pm 199.745$ & -20.7050311 & 0.00 \\
EE & $2063.15 \pm 165.955$ & $1980.60 \pm 202.501$ & -5.4626004 & 0.00 \\
\hline \hline
\end{tabular}


TABLE 4. Lipid profile and ratio between obese and non-obese

\begin{tabular}{lllll}
\hline \hline Variable & Obese & Non-obese & $\boldsymbol{t}$-value & $\boldsymbol{p}$-value \\
\hline TC & $190.18 \pm 55.025$ & $180.60 \pm 56.235$ & -2.1793365 & 0.0298 \\
HDL & $37.12 \pm 10.711$ & $35.75 \pm 10.404$ & -1.6571517 & 0.0982 \\
LDL & $118.78 \pm 44.576$ & $116.26 \pm 48.916$ & -0.6731205 & 0.5013 \\
TG & $200.03 \pm 136.845$ & $179.83 \pm 100.921$ & -2.2737985 & 0.0233 \\
Non-HDL & $153.06 \pm 53.707$ & $144.85 \pm 54.234$ & -1.9277592 & 0.0545 \\
TC: HDL & $5.49 \pm 2.085$ & $5.36 \pm 2.129$ & -0.7454256 & 0.4564 \\
LDL: HDL & $3.42 \pm 1.492$ & $3.45 \pm 1.587$ & 0.2647949 & 0.7913 \\
TG: HDL & $6.32 \pm 6.363$ & $5.85 \pm 5.631$ & -1.0141809 & 0.3110 \\
FBS & $6.60 \pm 2.318$ & $6.88 \pm 3.305$ & 1.1768081 & 0.2401 \\
IR & $3.46 \pm 2.689$ & $3.53 \pm 2.687$ & 0.3119236 & 0.7552 \\
\hline \hline
\end{tabular}

TABLE 5. The anthropometric data of the insulin resistance and insulin sensitive groups

\begin{tabular}{lllll}
\hline \hline Variable & $\mathbf{I R < 2}$ & $\mathbf{I R}>\mathbf{2}$ & $\boldsymbol{t}$-value & $\boldsymbol{p}$-value \\
\hline Age & $54.09 \pm 15.516$ & $52.60 \pm 14.444$ & 1.2915583 & 0.197 \\
Height & $159.34 \pm 6.536$ & $158.74 \pm 6.340$ & 1.2185308 & 0.223 \\
Weight & $63.36 \pm 7.036$ & $62.43 \pm 7.119$ & 1.7307617 & 0.084 \\
BMI & $26.22 \pm 2.569$ & $26.04 \pm 2.579$ & 0.9203194 & 0.357 \\
FBS & $5.21 \pm 0.844$ & $7.45 \pm 2.942$ & -15.8934368 & 0.00 \\
EI & $2098.81 \pm 223.788$ & $2086.50 \pm 241.592$ & 0.7015914 & 0.483 \\
EE & $2033.79 \pm 177.250$ & $2040.76 \pm 184.328$ & -0.5078884 & 0.611 \\
\hline \hline
\end{tabular}
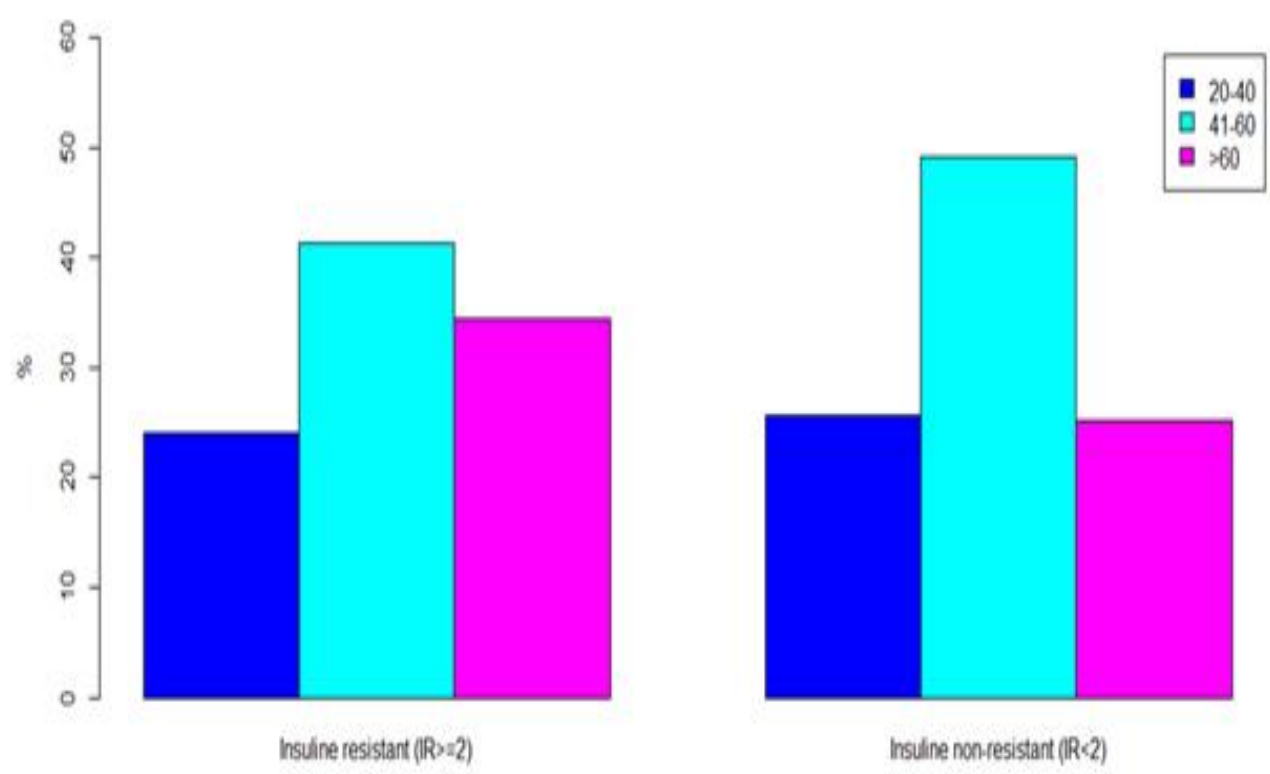

FIGURE 1 . Proportion of insulin resistance according to age groups (Group1-20-40yrs, Group 2-41 60yrs, Group3->60yrs)

Higher energy intake (2186.29 \pm 178.884$)$ relates higher risk of obesity ( $t$-value $3.312823 \mathrm{e}-65)$ than lower energy intake (1870.99 \pm 199.745$)$. The association between overweight and obese women (BMI $\geq 25 \mathrm{~kg} / \mathrm{m} 2)$ is more evident. High Energy Intake was associated with a modest increase in risk of obesity. Because BMI is a very strong risk factor for the development of T2D. We present associations between components of energy balance (EI \& EE) 
TABLE 6 . Lipid profile and ratio between insulin resistance \& sensitive group

\begin{tabular}{lllll}
\hline \hline Parameters & IR $<2$ & IR $>\mathbf{2}$ & $\boldsymbol{t}$-value & $\boldsymbol{p}$-value \\
\hline TC & $193.80 \pm 57.076$ & $174.66 \pm 50.319$ & -4.7572321 & 0.00 \\
HDL & $34.89 \pm 10.103$ & $40.16 \pm 10.814$ & 6.5247679 & 0.00 \\
LDL & $122.72 \pm 46.641$ & $108.93 \pm 43.175$ & -4.0731016 & 0.000053 \\
TG & $214.39 \pm 136.362$ & $154.77 \pm 97.037$ & -6.9762491 & 0.00 \\
TC: HDL & $5.90 \pm 2.151$ & $4.60 \pm 1.698$ & -9.1611265 & 0.00 \\
LDL: HDL & $3.71 \pm 1.510$ & $2.89 \pm 1.396$ & -7.4934685 & 0.00 \\
TG: HDL & $7.14 \pm 6.947$ & $4.33 \pm 3.585$ & -7.3821418 & 0.00 \\
\hline \hline
\end{tabular}

TABLE 7. ANOVA for age group and BMI

\begin{tabular}{lllll}
\hline \hline Age group & Differ & Lower & Upper & $\boldsymbol{p}_{\text {adj }}$ \\
\hline $41-60$ & 1.0100284 & 0.4190067 & 1.6010501 & 0.0001943 \\
$61-80$ & 0.1412650 & -0.3731842 & 0.6557141 & 0.7953485 \\
\hline \hline
\end{tabular}

stratified by BMI categories. We found significant difference on TG: HDL when regression analysis done for all lipid parameters. In South Asian women, obesity is associated with an adverse lipid profile. We find significantly higher TG and lower HDL-C level in obese than non-obese women. Likewise, the atherogenic Index (TC/HDL-C) is also significantly higher in obese than non-obese women. Significant differences are found in LDL, HDL, and TG between insulin resistance \& insulin sensitive groups. Among the ratios of lipid parameters in IR subjects, significant difference is found only in TG: HDL. However, BMI between age groups revealed that there is significant difference between two age groups. Energy gap estimation and nutrient intakes has been calculated from a self-report of food consumption. This survey has been done by using multiple telephone-administered. The nutrient data system for Research software (NDSR version 2012), licensed from the Nutritional Coordinating Center (NCC) at the University of Minnesota, was utilized to conduct the dietary interviews [17].

\section{DISCUSSION}

It suggests that there has been a significant reduction in energy expenditure over the last several decades. This reduction could be due to increased occupational automation, labour-saving devices associated with house work, and lifestyle choices for leisure time. Mathematical modeling was used to predict weight change since 1960 with predictions based on over $100 \mathrm{kcals} /$ day reduction estimated in occupational energy expenditure [11]. The results of this modeling demonstrate that reducing energy balance average $100 \mathrm{kcals} /$ day predicts closely the increase in body weight over the same period of time. An evaluation of the time spent in household activities by women over the last four decades indicates that time associated with household chores was reduced by at least seven hour/week with a concomitant decrease in energy expenditure of over $265 \mathrm{kcals} /$ day [18]. Not only are obesity and physical activity interrelated but they also positively affected insulin sensitivity and fat metabolism. They form more complex cluster of behavioural and metabolic phenotypes including diabetes and the metabolic syndrome. Energy flux, therefore has an impact on maintaining a stable weight. Preventing age-associated fitness loss decreases all-cause mortality regardless of weight change [19].

A recent review revealed a lack of long-term ( $>5$ years) data demonstrating the relationship between physical activity and non-communicable disease and dementia but 
concluded that physical activity has a positive influence on all these diseases [20]. Our study has several strengths. This population- based study represents from a wide range of socioeconomic backgrounds. The study has a low lost-to-follow-up rate. Detailed information on socioeconomic status and other lifestyle factors allowed for a comprehensive adjustment for these factors. The repeated dietary measurements in the study improved the quality of the dietary information. The high quality of data and the large sample size allowed us to comprehensively evaluate individual components and interactions of components of energy balanced in the etiology of T2D. If height, weight/BMI, energy intake and lower levels of PA were associated with diagnosis of T2D, an over-estimation of the association could also occur. There is also a need for structured exercise and physical activity level, both into account in the analysis, which should be subject to measurement error. In addition, a number of studies have also demonstrated that insulin resistance may be improved with regular exercise training even without calorie restriction or weight loss $[21,22,23]$.

\section{CONCLUSION}

There is also a need for an intervention study to evaluate small changes in the four variables of primary interest (weight, fatness, and energy intake and energy expenditure) using quarterly measurements for over 850 participants. We believe that there is a great need to evaluate the potential impact of teaching our children about energy balance that is how energy in food interacts with energy expenditure can improve an individual's metabolic profile without changing weight. A small change of energy balance can contribute for developing practical solutions to improve obesity crisis and public health.

\section{LIMITATIONS OF STUDY}

To determine the cause of metabolic disease and to prevent weight gain we need more emphasis on energy balance equation and interventional studies to obtain more conclusive test. There is a need of comparing and contrasting the beneficial effects of physical activity and body fitness. Also there is a need of large clinical studies to confirm this finding. Finally, we hope that within next decade we will be able to understand the pathophysiological link between metabolic diseases energy balance through the clinical approach.

\section{REFERENCES}

1. David G, Woods V, Li G, Buckle, P. The development of the Quick Exposure Check (QEC) for assessing exposure to risk factors for work-related musculoskeletal disorders. Applied Ergonomics. 2008; 39(1): 57-69.

DOI: $10.1016 /$ j.apergo.2007.03.002

2. Hamilton MT, Hamilton DG, Zderic TW. Exercise physiology versus inactivity physiology: An essential concept for understanding lipoprotein lipase regulation. Exercise and Sport Sciences Reviews. 2004; 32(4): 161-166.

DOI: $10.1097 / 00003677-200410000-00007$

3. Haskell WL, Lee IM, Pate RR, Powell KE, Blair SN, Franklin BA, .. Bauman A. Physical activity and public health: Updated recommendation for adults from the American college of sports medicine and the American heart association. Circulation. 2007; 39(8): 1423-1434. DOI: 10.1249/mss.0b013e3180616b27

4. Camoes M, Oliveira A, Lopes C. The role of physical activity and diet on overall and central obesity incidence. Journal of Physical Activity and Health. 2011; 8(6): 811-819. 
5. Villegas R, Shu XO, Yang G, Matthews CE, Li H, Cai H, . . Zheng W. Energy balance and type 2 diabetes: A report from the Shanghai women's health study. Nutrition, Metabolism and Cardiovascular Diseases. 2009; 19(3): 190-197. DOI: 10.1016/j.numecd.2008.06.003

6. Hand GA, Blair SN. Energy flux and its role in obesity and metabolic disease. US Endocrinol. 2014; 10(1): 59-63. DOI: 10.17925/USE.2014.10.01.59

7. MacLean PS, Higgins JA, Jackman MR, Johnson GC, Fleming-Elder BK, Wyatt HR, . . . Hill J. Peripheral metabolic responses to prolonged weight reduction that promote rapid, efficient regain in obesity-prone rats. American Journal of Physiology-Regulatory, Integrative and Comparative Physiology. 2006; 290(6): 1577-1588.

DOI: 10.1152 /ajpregu.00810.2005

8. Hill JO, Thompson H, Wyatt H. Weight maintenance: What's missing? Journal of the American Dietetic Association. 2005; 105(5): 63-66. DOI: 10.1016/j.jada.2005.02.016

9. Church TS, Thomas DM, Tudor-Locke C, Katzmarzyk PT, Earnest CP, Rodarte RQ., . . Bouchard C. Trends over 5 decades in U.S. occupation-related physical activity and their associations with obesity. PLoS One. 2011; 6(5): e19657. DOI: 10.1371/journal.pone.0019657

10. Hu FB, Li TY, Colditz GA, Willett WC, Manson, JE. Television watching and other sedentary behaviors in relation to risk of obesity and type 2 diabetes mellitus in women. JAMA. 2003; 289(14): 1785-1791. DOI: 10.1001/jama.289.14.1785

11. Thorp AA, McNaughton SA, Owen N, Dunstan DW. Independent and joint associations of TV viewing time and snack food consumption with the metabolic syndrome and its components; A cross-sectional study in Australian adults. International Journal of Behavioral Nutrition and Physical Activity. 2013; 10(96). DOI: 10.1186/1479-5868-10-96

12. Forbes GB, Brown MR, Welle SL, Lipinski BA. Deliberate overfeeding in women and men: Energy cost and composition of the weight gain. British Journal of Nutrition. 1986; 56(01): 1-9. DOI: 10.1079/BJN19860080

13. Swinburn BA, Sacks G, Lo, SK, Westerterp KR, Rush EC, Rosenbaum, M, . . Ravussin E. Estimating the changes in energy flux that characterize the rise in obesity prevalence; Estimating the changes in energy flux that characterize the rise in obesity prevalence. The American Journal of Clinical Nutrition. 2009; 89(6): 1723-1728. D0I: 10.3945/ajcn.2008.27061

14. King NA, Hopkins M, Caudwell P, Stubbs RJ, Blundell JE. Individual variability following 12 weeks of supervised exercise: Identification and characterization of compensation for exercise-induced weight loss. International Journal of Obesity. 2008; 32(1): 177-184. DOI: 10.1038/sj.ijo.0803712

15. Gallou-Kabani C, Junien C. Nutritional epigenomics of metabolic syndrome. Diabetes. 2005; 54(7): 1899-1906. DOI: 10.2337/diabetes.54.7.1899

16. Heagerty PJ, Comstock BA. Exploration of lagged associations using longitudinal data. Biometrics. 2013; 69(1): 197-205. DOI: $10.1111 / \mathrm{j} .1541-0420.2012 .01812 . x$

17. Novotny JA, Rumpler WVT, Judd OSEPH, Riddick H, Rhodes D, McDowell M, Briefel R. Diet interviews of subject pairs: How different persons recall eating the same foods. Journal of the American Dietetic Association. 2001; 101(10): 1189-1193. DOI: 10.1016/S0002-8223(01)00291-7

18. Archer E, Shook RP, Thomas DM, Church TS, Katzmarzyk PT, Hebert JR, . . Blair SN. 45-Year trends in women's use of time and household management energy expenditure. PLoS One. 2013; 8(2): e56620. D0I: 10.1371/journal.pone.0056620

19. Lee DC, Sui X, Artero EG, Lee IM, Church TS, McAuley PA, ... Blair SN. Long-term effects of changes in cardiorespiratory fitness and body mass index on all-cause and cardiovascular disease mortality in men: The aerobics center longitudinal study. Circulation. 2011; 124(23): 2483-2490. DOI: 10.1161/CIRCULATIONAHA.111.038422

20. Zhang P, Sui X, Hand GA, Hébert JR, Blair SN. Association of changes in fitness and body composition with cancer mortality in men. Medicine and Science in Sports and Exercise. 2014; 46(7): 1366-1374. D0I: 10.1249/MSS.0000000000000225

21. Cuff DJ, Meneilly GS, Martin A, Ignaszewski A, Tildesley HD, Frohlich JJ. Effective exercise modality to reduce insulin resistance in women with type 2 diabetes. Diabetes Care. 2003; 26(11): 2977-2982. DOI: 10.2337/diacare.26.11.2977 
22. Davis CL, Pollock NK, Waller JL, Allison JD, Dennis BA, Bassali R, . . Gower BA. Exercise dose and diabetes risk in overweight and obese children: A randomized controlled trial. JAMA. 2012. 308(11), 1103-1112.

DOI: 10.1001/2012.jama.10762

23. Lee S, Bacha F, Hannon T, Kuk JL, Boesch C, Arslanian S. Effects of aerobic versus resistance exercise without caloric restriction on abdominal fat, intrahepatic lipid, and insulin sensitivity in obese adolescent boys: A randomized, controlled trial. Diabetes. 2012; 61(11): 2787-2795. DOI: 10.2337/db12-0214

— This article does not have any appendix. - 